\title{
Malignant peritoneal mesothelioma in a patient with intestinal fistula, incisional hernia and abdominal infection: A case report
}

\author{
SEN HONG ${ }^{1 *}$, MIAO-MIAO BI ${ }^{2 *}$, PING-WEI ZHAO ${ }^{1}, \mathrm{XU}_{\text {WANG }}{ }^{1}$, \\ QING-YANG KONG ${ }^{3}$, YONG-TAO WANG ${ }^{1}$ and LEI WANG ${ }^{1}$ \\ ${ }^{1}$ Department of Colon and Anal Surgery, The First Hospital of Jilin University, Changchun, Jilin 130021; \\ ${ }^{2}$ Department of Ophthalmology, The China-Japan Union Hospital of Jilin University, Changchun, Jilin 130033; \\ ${ }^{3}$ Department of Pathology, The First Hospital of Jilin University, Changchun, Jilin 130021 P.R. China
}

Received December 9, 2014; Accepted January 11, 2016

DOI: $10.3892 / 01.2016 .4203$

\begin{abstract}
Malignant mesothelioma is a rare type of cancer, most commonly associated with exposure to asbestos. Mesothelioma of the peritoneum, the membrane lining the abdominal cavity, is extremely rare. The current study reports the case of a 60 -year-old female who presented with intestinal fistula, recurrent incisional hernia and abdominal infection, with no history of asbestos exposure, and was diagnosed with clear cell MPM. Computed tomography scans of the abdomen revealed extensive small bowel adhesions and massive peritoneal effusion. Histological examination of biopsy specimens indicated a diagnosis of malignant peritoneal mesothelioma with clear cell morphology. A laparotomy was performed, with subsequent resection of the bowel with fistula. Follow-up examination performed at 1-year post-surgery revealed that the patient was alive and in generally good health.
\end{abstract}

\section{Introduction}

Mesothelioma is a rare type of cancer originating from the surface linings of serous cavities; these membranes include the pleura, peritoneum and pericardium $(1,2)$. Malignant peritoneal mesothelioma (MPM) is highly aggressive, accounting for $25 \%$ of all mesothelioma cases (3), and is the second most common mesothelioma subtype after pleural mesothelioma. Mesothelioma exhibits a predilection for males with a history of work-related asbestos exposure (2).

The four main cellular subtypes of mesothelioma are epithelioid, sarcomatoid, biphasic and desmoplastic (4,5). Of these subtypes, epithelioid mesothelioma is the most common.

Correspondence to: Dr Lei Wang, Department of Colon and Anal Surgery, The First Hospital of Jilin University, 3302 Jilin Road, Changchun, Jilin 130021, P.R. China

E-mail: wanglei-md@sohu.com

${ }^{*}$ Contributed equally

Key words: malignant, peritoneal mesothelioma, clear cell type
Cells of epithelioid mesothelioma tumors are usually epithelial-like and may be cuboidal, tubular or flattened. In rare cases, tumors may exhibit deciduoid, pleomorphic, signet-ring or clear cell features (6). Clear cell-type mesothelioma is extremely rare, with only a few individual case reports published previously $(1,6-8)$. In the present study, a rare case of clear cell MPM is reported in a 60-year-old female with no history of asbestos exposure.

\section{Case report}

On August 21, 2012, a 60-year-old female was admitted to The First Hospital of Jilin University (Changchun, China) with intestinal fistula, incisional hernia and abdominal infection. The patient had undergone a cesarean section and appendectomy 30 years previously, and the resultant abdominal incision had healed well. In August 2008, the patient presented to a local hospital (Central Hospital of Siping, Siping, China) with an incisional hernia located at the cesarean section incision. Incisional hernia repair surgery was subsequently performed, and the section of the small bowel in the incisional sac was returned to the abdominal cavity. In April 2011, the patient presented again with an incisional hernia with a protruded abdominal mass. During a laparotomy, a massive cystic cavity with purulence outside of the peritoneum in the lower abdomen was found. The purulent cyst was removed, the patient's general condition improved and the abdominal mass disappeared.

In August 2012, the patient was admitted to a local hospital for the third time due to the recurrence of the incisional hernia with an abdominal mass, which exuded yellowish fluid. A secondary laparotomy was performed with the same findings as in 2011. Following aspiration of the purulent fluid, a drainage tube was inserted into the incisional hernia lesion. At 3 days post-surgery, fecal-like materials passed through the drainage tube, and the patient was transferred to The First Hospital of Jilin University for further management. The patient had no history of smoking or alcohol consumption, and no history of asbestos exposure.

A physical examination revealed abdominal distension with ascites, but no abdominal pain, stiffness or rebound tenderness. Laboratory blood examinations revealed a 
marginal increase in platelet number $\left(381 \times 10^{9}\right.$ cells $/ 1$; normal range, 100-300 $\times 10^{9}$ cells/1). Red and white blood cell count and liver and kidney functions were normal. Chest and plain abdominal radiographs were normal. Extensive small bowel adhesion and massive peritoneal effusion were observed on the abdominal computed tomography scans (Fig. 1). The patient was admitted for further management of the intestinal fistula, incisional hernia and abdominal infection.

A laparotomy was performed on August 27, 2012. A massive purulent cavity $(15 \times 12 \times 3 \mathrm{~cm})$ was identified outside the peritoneum in the lower abdomen. Multiple white nodules of variable size $(1-5 \mathrm{~mm})$ were found in the peritoneum. Extensive bowel adhesions were also observed. No significant enlargement of the lymph nodes was identified, and no lesions were found in the liver, pancreas, spleen, kidneys or ovaries. The adhesive bowels were separated. An intestinal fistula was identified $\sim 80 \mathrm{~cm}$ from the distal end of the jejunum suspensory ligament, closely connected to the cranial end of the purulent cavity. The necrotic intestinal fistula was resected and multiple white tumor-like nodules were observed on the surface (Fig. 2).

Histological examination of the intestinal fistula biopsy specimens indicated the presence of MPM, with invasion to the intestinal submucosa and mesenterium (Fig. 3). Positive immunoreactivity was observed for calretinin (Fig. 3D), pan-cytokeratin (Fig. 3E), cytokeratin 7, cytokeratin 14, cytokeratin 19, epithelial membrane antigen (EMA; Fig. 3F), cluster of differentiation (CD)99, caldesmon and Ki-67. Negative expression of calponin, S-100, vimentin, CD34, HMB-45, smooth muscle actin, desmin, CD117 and DOG1 proteins was also observed. A fistula of the anastomotic lesion occurred 1 week post-operatively. Local flushing and dressing of the lesions was performed. No further medical treatment was administered. Follow-up examination 1 year after surgery revealed that the patient was in generally good health, and at the time of writing, the patient remained alive with no signs of recurrence.

\section{Discussion}

MPM is a rare disease with varied and non-specific clinical presentations, and thus its diagnosis is often overlooked by surgeons and pathologists (2). The patient in the present report underwent three laparotomies as a result of incisional hernia and abdominal infection. However, only after the last pathological analysis was the presence of MPM diagnosed. Increased awareness and caution with regard to this disease is therefore required.

A history of asbestos exposure is closely associated with the susceptibility to MPM (9). The incidence of MPM is higher in males than females, which may be related to their higher risk of occupational exposure to asbestos (10). The proportion of patients with mesothelioma reporting histories of asbestos exposure ranged from 5-83\% (9). However, the absence of a history of asbestos exposure does not exclude the possibility of malignant mesothelioma. Other risk factors of peritoneal mesothelioma include recurrent peritonitis (11), radiation therapy (12), mica exposure (13), and thorium dioxide administration for cholangiography (14). Notably, the patient in the present report had no history of asbestos exposure.

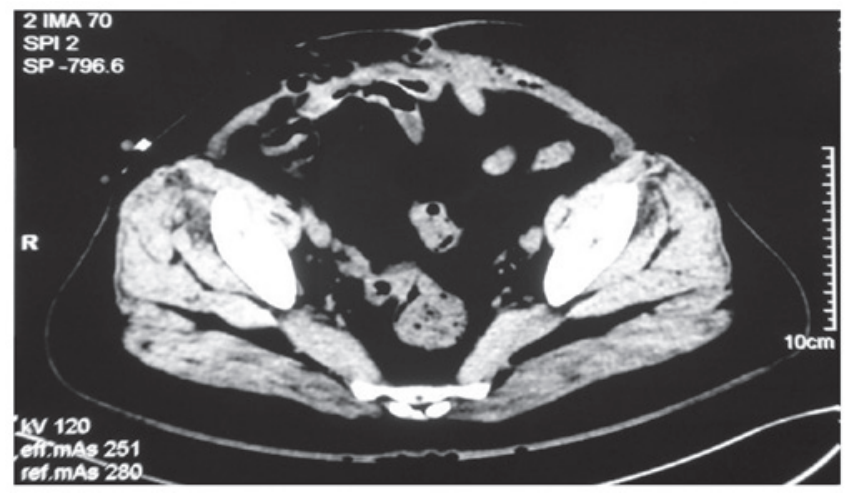

Figure 1. Abdominal computed tomography scan revealing extensive small bowel adhesion and massive abdominal ascites.

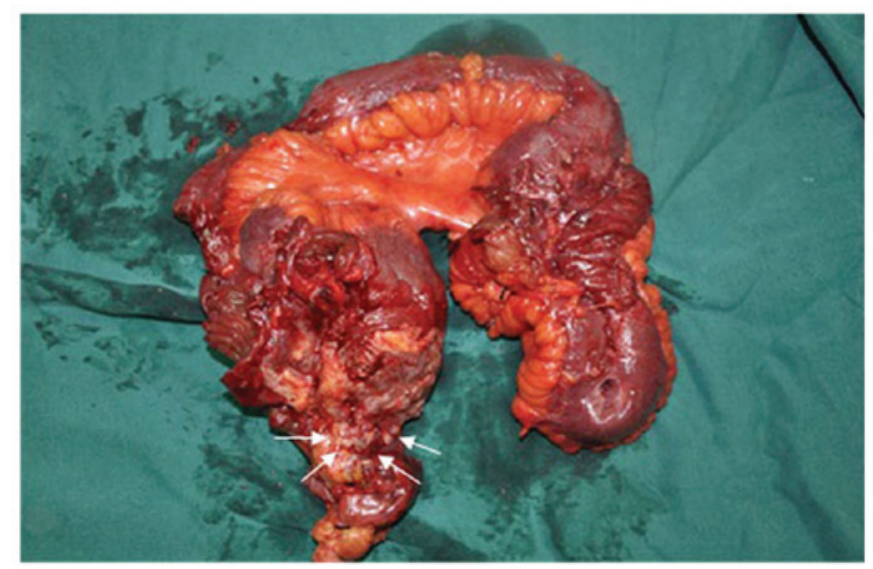

Figure 2. Resected necrotic intestinal fistula lesion. Arrows indicate multiple tumor nodules.

Clinical presentations of MPM are non-specific. Abdominal distention and the identification of an abdominal mass are the most common primary presentations (1). Approximately $70 \%$ of patients exhibit serous ascetic fluid, which is produced by tumor nodules (3). Thrombocytosis is another common symptom observed in MPM patients (15), occurring in 40-80\% of cases $(9,16)$. In the present case, routine blood tests revealed a marginal increase in platelet count $\left(381 \times 10^{9}\right.$ cells/l), which may have been due to the presence of tumor cells. However, the exact pathogenesis of thrombocytosis remains to be elucidated.

The most specific manifestation of MPM identified during laparoscopy or laparotomy is the presence of diffuse whitish tumor nodules on the peritoneum $(2,3)$. Aggregation of these nodules to form masses or plaques may occur. The MPM patient in the current report presented with an intestinal fistula, incisional hernia and abdominal infection, which to the best of our knowledge, has not been reported previously in the literature. However, the coexistence of inguinal and umbilical hernia and MPM is more common $(4,9,17,18)$. Munkholm-Larsen et al (3) reported that $13 \%$ of MPM patients exhibit a newly developed abdominal wall hernia. The development of a hernia in MPM patients may be due to the accumulation of intra-abdominal pressure from ascites. However, the presence of MPM in the hernial sac may be the result of chronic inflammation (9). Recurrent or chronic infection as a main presentation of the disease 

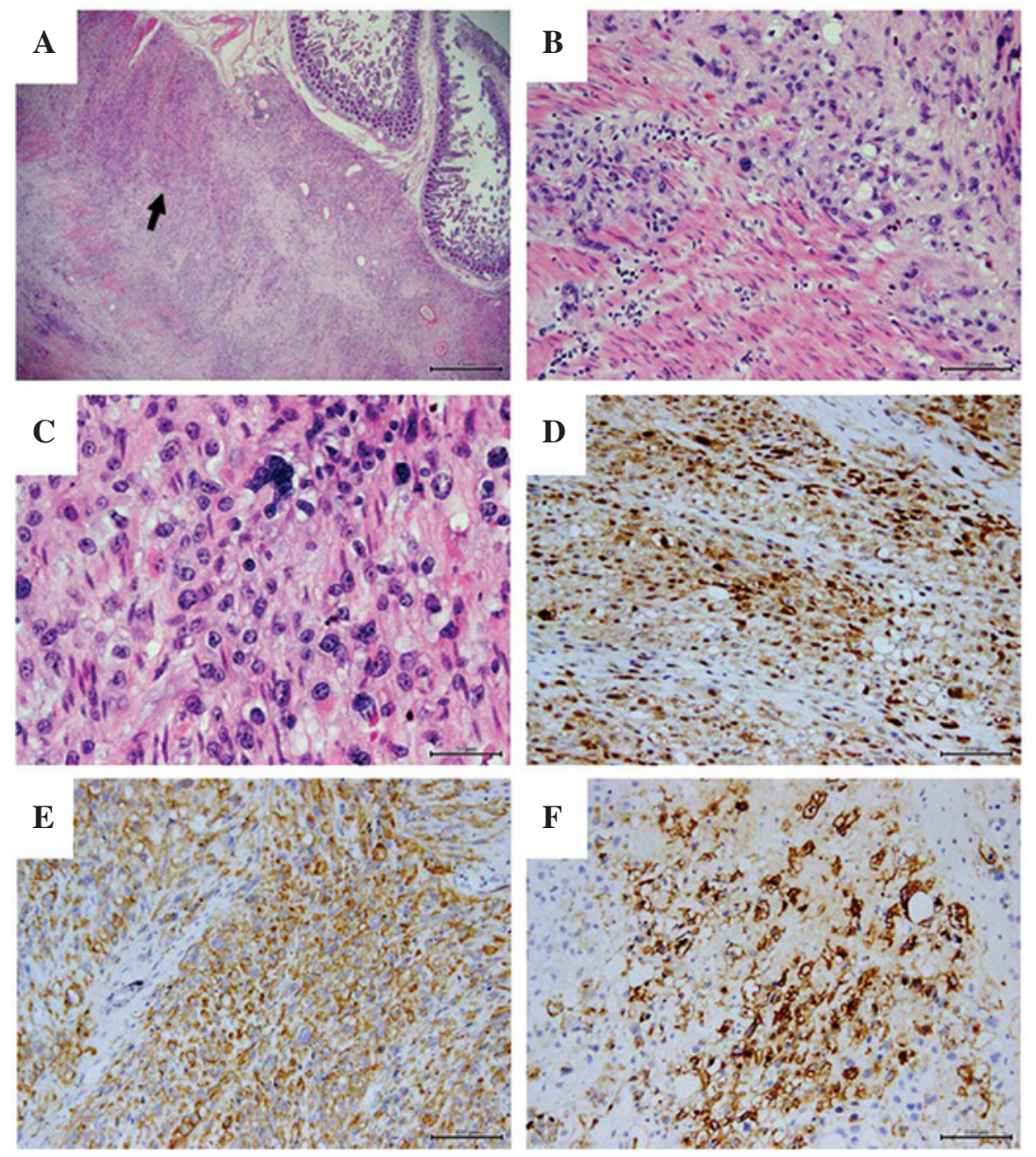

Figure 3. Histopathological and immunoreactive features of malignant peritoneal mesothelioma. (A) Mesothelioma with epithelial-like cells (indicated by the black arrow) and intestinal mucosa (stain, H\&E; scale bar, $1 \mathrm{~mm}$ ). (B) Micrograph of mesothelioma with epithelial-like cells and clear cells (stain, H\&E; scale bar, $100 \mu \mathrm{m}$ ). (C) Clear cells with prominent nuclei and clear cytoplasm in tumor cells (stain, H\&E; scale bar, $50 \mu \mathrm{m}$ ). (D) Diffuse positive immunoreactivity for calretinin in nuclei and cytoplasm (scale, $100 \mu \mathrm{m}$ ). (E) The majority of the tumor was pan-cytokeratin-positive (scale bar, $100 \mu \mathrm{m}$ ). (F) The majority of cellular membranes were epithelial membrane antigen-positive (scale bar, $100 \mu \mathrm{m}$ ). $\mathrm{H} \& \mathrm{E}$, hematoxylin and eosin.

is not that common in MPM patients, although certain patients may exhibit symptoms of inflammatory bowel disease $(19,20)$. Peritoneal mesothelioma complicated with a fistula is rare, with only a few cases reported previously; Govender et al reported the occurrence of a colojejunal fistula and a long sigmoid stricture in a 53-year-old male with peritoneal mesothelioma (21), and McCaffrey et al (22) reported an unusual case of benign peritoneal mesothelioma in a 59-year-old female with colovesical fistula and bilateral hydronephrosis formation. We hypothesize that the occurrence of fistulae may be due to tissue necrosis, chronic stimulation of local inflammation and a lack of blood supply to the intestinal tract.

The diagnosis of MPM is difficult to establish pre-operatively. Usually, the diagnosis is based on pathological hematoxylin-eosin staining and immunohistochemical staining from biopsy samples collected during laparoscopy and laparotomy (3). Due to the high prevalence of ascites in MPM patients, the cytological examination of ascetic fluid is often required. However, the results of ascites cytology are often non-specific (23). Cytological examination was not performed in the present study. Microscopic examination of the intestinal fistula biopsy specimen revealed the presence of undifferentiated malignant neoplastic nodules with infiltration of the intestinal submucosa and mesenterium (Fig. 3A).
These epithelial-like cells were diverse in size and shape, with a number of clear cells exhibiting prominent nuclei and clear cytoplasm (Fig. 3B and C). Malignant mesothelioma commonly exhibit positive immunoreactivity for calretinin, EMA, Wilms' tumor 1 antigen, serum mesothelin-related protein, podoplanin, cytokeratin (CK)5/6, D2-40 antibodies, CA12-5, CA15-3, hyaluronic acid, osteopontin and pan-CK, and exhibit negative immunoreactivity for calponin, S-100, vimentin, HMB-45, CD117, DOG1, Ber-EP4, MOC-31, TAG72, CA19-9, CD15, monoclonal carcinoembryonic antigen and BG-8 antibodies (24).

The differential diagnosis of MPM is quite broad, and also depends on histological analyses (25). The main diagnostic difficulty is differentiating MPM from adenocarcinoma (2). Among the aforementioned markers, calretinin, a vitamin D-dependent calcium-binding protein, is the most definitive in differentiating mesothelioma from adenocarcinoma (26); calretinin is largely present in mesothelioma, but absent in adenocarcinoma. Renal cell carcinoma may also complicate the diagnosis of MPM; calretinin and cytokeratin are positively expressed in epithelioid mesothelioma, but not in renal cell carcinoma (8). Strong EMA expression and negative desmin expression also indicate malignant mesothelioma, and may be used as reliable markers to distinguish malignant mesothelioma from benign reactive 
mesothelial proliferation $(2,25,27)$. The positive immunostaining of cytokeratin also aids to distinguish malignant mesothelioma from melanoma and sarcoma, and also confirms tumor invasion (1). In the present case, the macroscopic observations during laparotomy, the microscopic morphologies, and the positive immunoreactivity of several markers indicated the diagnosis of MPM.

At present, no optimal treatment strategy for MPM has been established. Due to the rarity of this tumor, few previous studies have investigated possible treatment regimens. Eltabbakh et al (28) demonstrated that cytoreduction surgery combined with chemotherapy (paclitaxel and cisplatin) may provide a benefit for female patients. Sugarbaker et al (29) also confirmed that a combined regimen of cytoreduction surgery and intraperitoneal chemotherapy prolonged survival and completely resolved ascetic fluid in the majority of patients. However, the distribution of tumor nodules is not limited to the peritoneal surface, and may also extend to the small bowel and mesentery, which complicates cytoreduction surgery, preventing completion of the procedure in certain cases.

The prognosis of patients with MPM is relatively poor, with a reported survival time of $\sim 1$ year following the diagnosis $(2,28)$. In one study, the median survival time of diffuse MPM patients improved to 92 months, with a 5-year survival rate of 59\%, following combined treatment with cytoreductive surgery and intraperitoneal chemotherapy (30). However, the patient in the present case refused all chemotherapy. Despite this, the patient survived, was in generally good health at the follow-up examination performed at 1 year post-surgery and remains alive at the time of writing this study.

MPM is a rare disease, and thus information regarding this type of cancer remains limited. The present case study highlights that a diagnosis of MPM should be considered in patients with intractable intestinal fistula, incisional hernia and abdominal infection. Stimulation due to recurrent inflammation may facilitate tumor progression and compromise the healing of an incisional hernia. Furthermore, presentations of incisional hernia with intestinal fistula accompanied by peritoneal infection may obscure recognition of malignant mesothelioma, and make an accurate diagnosis of MPM difficult.

\section{References}

1. Dessy E, Falleni M, Braidotti P, Del Curto B, Panigalli T and Pietra GG: Unusual clear cell variant of epithelioid mesothelioma. Arch Pathol Lab Med 125: 1588-1590, 2001.

2. Robinson BW and Lake RA: Advances in malignant mesothelioma. N Engl J Med 353: 1591-1603, 2005.

3. Munkholm-Larsen S, Cao CQ and Yan TD: Malignant peritoneal mesothelioma. World J Gastrointest Surg 1: 38-48, 2009.

4. D'Ambrosio V: Mesothelioma as primary tumor of the peritoneum. A case report. J Med Soc N J 70: 637-639, 1973.

5. Hammar SP: Macroscopic, histologic, histochemical, immunohistochemical, and ultrastructural features of mesothelioma. Ultrastruct Pathol 30: 3-17, 2006.

6. Gkogkou C, Samitas K and Foteinou M: Primary pleural epithelioid mesothelioma of clear cell type: A case report and review of current literature. Ultrastruct Pathol 35: 267-270, 2011.

7. Ordóñez NG: Mesothelioma with clear cell features: An ultrastructural and immunohistochemical study of 20 cases. Hum Pathol 36: 465-473, 2005.
8. Ordóñez NG: Clear cell mesothelioma presenting as an incarcerated abdominal hernia. Virchows Arch 447: 823-827, 2005.

9. de Pangher Manzini V: Malignant peritoneal mesothelioma. Tumori 91: 1-5, 2005.

10. Sugarbaker PH, Welch LS, Mohamed F and Glehen O: A review of peritoneal mesothelioma at the washington cancer institute. Surg Oncol Clin N Am 12: 605-621, 2003.

11. Riddell RH, Goodman MJ and Moossa AR: Peritoneal malignant mesothelioma in a patient with recurrent peritonitis. Cancer 48: 134-139, 1981.

12. Antman KH, Corson JM, Li FP, Greenberger J, Sytkowski A, Henson DE and Weinstein L: Malignant mesothelioma following radiation exposure. J Clin Oncol 1: 695-700, 1983.

13. Chahinian AP, Pajak TF, Holland JF, Norton L, Ambinder RM and Mandel EM: Diffuse malignant mesothelioma. Prospective evaluation of 69 patients. Ann Intern Med 96: 746-755, 1982.

14. Maurer R and Egloff B: Malignant peritoneal mesothelioma after cholangiography with thorotrast. Cancer 36: 1381-1385, 1975.

15. De Pangher Manzini V, Brollo A and Bianchi C: Thrombocytosis in malignant pleural mesothelioma. Tumori 76: 576-578, 1990.

16. Piccigallo E, Jeffers LJ, Reddy KR, Caldironi MW, Parenti A and Schiff ER: Malignant peritoneal mesothelioma. A clinical and laparoscopic study of ten cases. Dig Dis Sci 33: 633-639, 1988.

17. Mirabella F: Peritoneal mesothelioma and abdominal hernias. Minerva Med 87: 21-24, 1996 (In Italian).

18. Smith TR: Malignant peritoneal mesothelioma: Marked variability of CT findings. Abdom Imaging 19: 27-29, 1994.

19. Higashihara M, Sunaga S, Tange T, Oohashi H and Kurokawa K: Increased secretion of interleukin-6 in malignant mesothelioma cells from a patient with marked thrombocytosis. Cancer 70: 2105-2108, 1992.

20. Melero M, Lloveras J, Waisman H, Elsner B and Baldessari E: Malignant peritoneal mesothelioma. An infrequent cause of prolonged fever syndrome and leucocytosis in a young adult. Medicina (B Aires) 55: 48-50, 1995 (In Spanish).

21. Govender SS, Seebaran AR, Moodley V and Rajput MC: Peritoneal mesothelioma complicated by colojejunal fistula. A case report. S Afr J Surg 25: 103-105, 1987.

22. McCaffrey JC, Foo FJ, Dalal N and Siddiqui KH: Benign multicystic peritoneal mesothelioma associated with hydronephrosis and colovesical fistula formation: Report of a case. Tumori 95: 808-810, 2009.

23. Yu GH, Soma L, Hahn S and Friedberg JS: Changing clinical course of patients with malignant mesothelioma: Implications for FNA cytology and utility of immunocytochemical staining. Diagn Cytopathol 24: 322-327, 2001.

24. Ahmed I, Ahmed Tipu S and Ishtiaq S: Malignant mesothelioma. Pak J Med Sci 29: 1433-1438, 2013 (Review).

25. Baker PM, Clement PB and Young RH: Malignant peritoneal mesothelioma in women: A study of 75 cases with emphasis on their morphologic spectrum and differential diagnosis. Am J Clin Pathol 123: 724-737, 2005.

26. Chhieng DC, Yee H, Schaefer D, Cangiarella JF, Jagirdar J, Chiriboga LA, Jagirdar J, Chiriboga LA and Cohen JM: Calretinin staining pattern aids in the differentiation of mesothelioma from adenocarcinoma in serous effusions. Cancer 90: 194-200, 2000

27. Saad RS, Cho P, Liu YL and Silverman JF: The value of epithelial membrane antigen expression in separating benign mesothelial proliferation from malignant mesothelioma: A comparative study. Diagn Cytopathol 32: 156-159, 2005.

28. Eltabbakh GH, Piver MS, Hempling RE, Recio FO and Intengen ME: Clinical picture, response to therapy, and survival of women with diffuse malignant peritoneal mesothelioma. J Surg Oncol 70: 6-12, 1999.

29. Sugarbaker PH, Yan TD, Stuart OA and Yoo D: Comprehensive management of diffuse malignant peritoneal mesothelioma. Eur J Surg Oncol 32: 686-691, 2006.

30. Feldman AL, Libutti SK, Pingpank JF, Bartlett DL, Beresnev TH, Mavroukakis SM, Steinberg SM, Liewehr DJ, Kleiner DE and Alexander HR: Analysis of factors associated with outcome in patients with malignant peritoneal mesothelioma undergoing surgical debulking and intraperitoneal chemotherapy. J Clin Oncol 21: 4560-4567, 2003. 